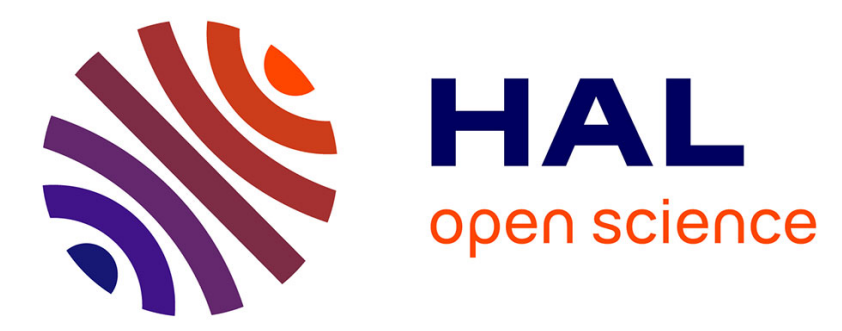

\title{
Comparison and validation of Oomycetes metabarcoding primers for Phytophthora high throughput sequencing
}

Jean Legeay, Claude Husson, Tristan Cordier, Corinne Vacher, Benoit Marçais, Marc Buee

\section{- To cite this version:}

Jean Legeay, Claude Husson, Tristan Cordier, Corinne Vacher, Benoit Marçais, et al.. Comparison and validation of Oomycetes metabarcoding primers for Phytophthora high throughput sequencing. Journal of Plant Pathology, 2019, 101 (3), pp.743-748. 10.1007/s42161-019-00276-9 . hal-02618173

\section{HAL Id: hal-02618173 \\ https://hal.inrae.fr/hal-02618173}

Submitted on 11 Dec 2020

HAL is a multi-disciplinary open access archive for the deposit and dissemination of scientific research documents, whether they are published or not. The documents may come from teaching and research institutions in France or abroad, or from public or private research centers.
L'archive ouverte pluridisciplinaire HAL, est destinée au dépôt et à la diffusion de documents scientifiques de niveau recherche, publiés ou non, émanant des établissements d'enseignement et de recherche français ou étrangers, des laboratoires publics ou privés. 
Journal of Plant Pathology, 2019, 101: 743-748

Doi: $10.1007 / \mathrm{s} 42161-019-00276-9$

hal-02618173

Online version of this article (https://doi.org/10.1007/s42161-019-00276-9) contains supplementary material, which is available to authorized users.

Comparison and validation of Oomycetes metabarcoding primers for Phytophthora high throughput sequencing

\author{
Jean Legeay ${ }^{1}$, Claude Husson ${ }^{1,2}$, Tristan Cordier $^{3}$, Corinne Vacher ${ }^{3}$, \\ Benoît Marcais ${ }^{1}$, Marc Buée ${ }^{1}$
}

1 Université de Lorraine, Inra, IAM , F-54000 Nancy, France,

2 DGAL, SDQPV, Département de la santé des forêts, Ministère de l'agriculture et de l'alimentation, 75015 Paris, France

${ }^{3}$ BIOGECO, INRA, University of Bordeaux, 33615 Pessac, France

\title{
Abstract
}

Oomycetes are eukaryotic plant pathogens that require health monitoring. Highthroughput sequencing (HTS) methods replace progressively cultivation-based approaches in soil surveys of Oomycetes, but very little control has been done from synthetic communities. Indeed, several potential biases do exist and need to be assessed for Oomycetes communities. We created a mock community by mixing DNA from 24 Phytophthora species. We amplified two barcode regions with Oomycete-specific primers before HTS. With this aim, we used three primer sets in nested PCR amplification, targeting the ITS-1 region or the RAS gene region. The three nested PCR strategies proved to be a reliable qualitative approach, identifying approximately $95 \%$ of the species after Illumina Miseq sequencing and bioinformatic analysis. However, quantitative proportions of each species showed distortions compared to the original mixture of the mock. In addition, we compared the two ITS primer sets on soil environmental DNA sampled from temperate forests. The ' oom18S-ITS7/18ph2f-5.8S-1R ' primer set, more specific to Phytophthora, was able to detect seven Phytophthora species, confirming what was expected for temperate forests. Using the 'DC6-ITS7/oom18S-ITS7' primer set that covers the broader Peronosporaceans, we detected only one Phytophthora species among the dominance of Pythium and Phytopythium species. We concluded that 'oom18SITS7/18ph2f-5.8S-1R ' primer set is a reliable tool for the qualitative description of environmental Phytophthora communities.

Keywords: Phytophthora. Metabarcoding. ITS region. RAS gene. Mock community 
Oomycetes are fungus-like eukaryotic organisms, including widespread plant pathogens such as Phytophthora infestans, P. ramorum or P. cinnamomi (Hansen 2008; Kamoun et al. 2015). Many species of Phytophthora have co-evolved with their hosts in their native ecosystems and do not cause severe diseases in those environmental conditions. However, when introduced in a new environment, they may become invasive and represent important threats to the managed or natural ecosystems (Hansen 2008). Examples include $P$. ramorum, $P$. uniformis or $P$. lateralis which are important invasive pathogens in Europe and/or North America but do not appear to be significant pathogens in their native environments (Brasier et al. 2010; Aguayo et al. 2013; Turner et al. 2017). It is thus important to increase our knowledge of Phytophthora communities, in particular of species that do not cause conspicuous damages to their host and metabarcoding of environmental DNA represents a new valuable tool for this purpose.

Indeed, DNA metabarcoding and high throughput sequencing (HTS) have been used for addressing significant questions in ecology and biodiversity assessment (Taberlet et al. 2012). Recently, these culture-independent approaches have been applied to Oomycete communities, skipping the traditional steps of baiting on trap leaves, and isolation on Petri dish, and increasing the rate of data acquisition. Sequencing of Internal Transcribed Spacer (ITS) regions is the universal barcode for fungal diversity analysis (Schoch et al. 2012). This molecular marker has been used for Oomycetes also (Coince et al. 2013, Vannini et al. 2013; Sapkota and Nicolaisen 2015; Riit et al. 2016). Similar to the diversity of fungal primer pairs (Bellemain et al. 2010; Tedersoo et al. 2015), oomycetes communities have been investigated thanks different primer pairs without any validation from artificial mock. Interestingly, few studies focused on Phytophthora communities using the 18Ph2F- 5.8S-1R primer pair (Scibetta et al. 2012; Català et al. 2015). Nevertheless, metabarcoding revealed multiple biases, including sequence errors during PCR, library preparation, primer template mismatches bias or primer specificity (Tedersoo et al. 2018). In recent studies focusing on the Phytophthora community, very little control has been done, needing a benchmarking effort for this ecological group. Mock communities have proved to be efficient for revealing potential biases involved along the methodological steps of metabarcoding studies targeting fun gal or protists communities (Bokulich et al. 2013; Egge et al. 2013; Taylor et al. 2016). Artificial communities are a particularly efficient tool to reveal mismatches between the read abundance and the initial proportion of species. To our knowledge, no study has used it yet for Phytophthora community studies. The aim of this study was to create mock communities of Phytophthora species, covering the nine most important clades, then to compare our three sets of primers targeting Oomycete sub-groups and validate some of them as tools for studying Phytophthora communities by HTS.

Mock communities were created from genomic DNAs of 24 Phytophthora species (Table 1) with or without back ground metagenomic DNAs from other microorganisms (Table S1). This additional microbial DNA (fungal or bacterial) aimed 
to control the potential mismatch errors between Phytophthora DNA and other microbial DNA. The different mock communities were amplified using three sets of primers, targeting the Phytophthora genus at the ITS or RAS-Ypt loci, or the broader Peronosporaceae group at the ITS locus (Table 2). The two ITS sets of primers were also tested on environmental samples collected from forests of NE France.

Table 1. Phytophthora isolates used in the mock community. ITS sequences were deposited in GenBank under accession Nos. MH178327-MH178350. The sequences of RAS-Ypt regions have been deposited in the DRYAD database (http://datadryad.org/). DRYAD entry https://doi.org/10.5061/dryad.68tk49

\begin{tabular}{llc}
\hline Name & Species & Clade \\
\hline PAU60 & Phytophthora uniformis & 7 \\
PAM54 & Phytophthora xmultiformis & 7 \\
Pc1A21 & Phytophthora xcambivora & 7 \\
87_neb & Phytophthora cinnamomi & 6 \\
CIN4 & Phytophthora cinnamomi & 6 \\
BBA65909 & Phytophthora cryptogea & 8 \\
2KE4 & Phytophthora europaea & 7 \\
PFF CSL & Phytophthora fragariae & 7 \\
gon1 & Phytophthora gonapodyides & 6 \\
lat1 & Phytophthora lateralis & 8 \\
PP2 & Phytophthora parasitica & 1 \\
cit2 & Phytophthora pini & 2 \\
resi1 & Phytophthora plurivora & 2 \\
resi32 & Phytophthora pseudosyringae & 3 \\
2KP7 & Phytophthora quercina & $\mathrm{NA}$ \\
ram1 & Phytophthora ramorum & 8 \\
soj1 & Phytophthora sojae & 7 \\
resi73 & Phytophthora syringae & 8 \\
Plac3 & Phytophthora lacustris & 6 \\
27_neb & Phytophthora heveae & 5 \\
1288_neb & Phytophthora castaneae & 5 \\
1543_neb & Phytophthora sp. neb_1543 & 9 \\
resi89 & Phytophthora sp. hungarica & 6 \\
resi51 & Phytophthora obrutafolium & 6 \\
NII72-5 & Phytophthora infestans & 1 \\
\hline
\end{tabular}

Genomic DNA was extracted from 25 Phytophthora sp. isolates (INRA Nancy collection) corresponding to 24 species, then quantified with a Qubit $\circledast 2.0$ Fluorometer (Life technologies) at $30 \mathrm{ng}$ in $2 \mu \mathrm{l}$. Bacterial and eukaryotic genomic DNA used as background, was extracted from 306 isolates (Table S1) from INRA collections. Additionally, eight environmental samples were obtained from soils collected in April 2016 at the base of four trees each (four soil samples per tree from 5 to $10 \mathrm{~cm}$ depth) in two forest plots of north-eastern France. The sampled trees belonged to Alnus glutinosa, Quercus sp., Fraxinus excelsior and Carpinus betulus. Environmental DNA was extracted with the Fast DNA SPIN Kit for soil (MP Biomedicals, Solon, $\mathrm{OH}, \mathrm{USA}$ ) according to the manufacturer' $s$ instructions. 
Three amplicon libraries (hereafter referred to as PERO_ITS, PHYTO_ITS and PHYTO_RAS) were produced using different combinations of primers. The PERO_ITS library (targeting Peronosporaceae) that has resulted from a nested PCR amplification of the ITS1 and 18S region fragment, used first the DC6 - ITS7 primers (Cooke et al. 2000) and then the newly designed primer pair Oom18s - ITS7 (Fig. 1; Table 2). For the PHYTO_ITS library (targeting Phytophthora), the ITS1 was amplified by another nested PCR approach, using first the Oom18s - ITS7 primers pair and then the Phytophthora-specific primer pair 18Ph2F-5.8S-1R (Scibetta et al. 2012). For both ITS libraries, $2 \mu$ I of the first-round amplicons diluted 10 times was used as DNA sample for the second round following the same PCR conditions. The second PCR round was conducted in triplicate, the amplicons being pooled and purified afterward. Finally, the PHYTO_RAS library (targeting Phytophthora, on the RAS-Ypt gene) was produced as described by Weir et al. (2015). For each PCR assay, $2 \mu$ I of DNA material were added to $18 \mu$ I of PCR reactive solution. Detailed temperature and duration for each PCR cycle are provided in Table S2. Multiplexing and Illumina MiSeq sequencing (Reagent Kit v3 chemistry, 2x250pb) were done by the GeT-PlaGe platform of Toulouse (http://get.genotoul.fr/).

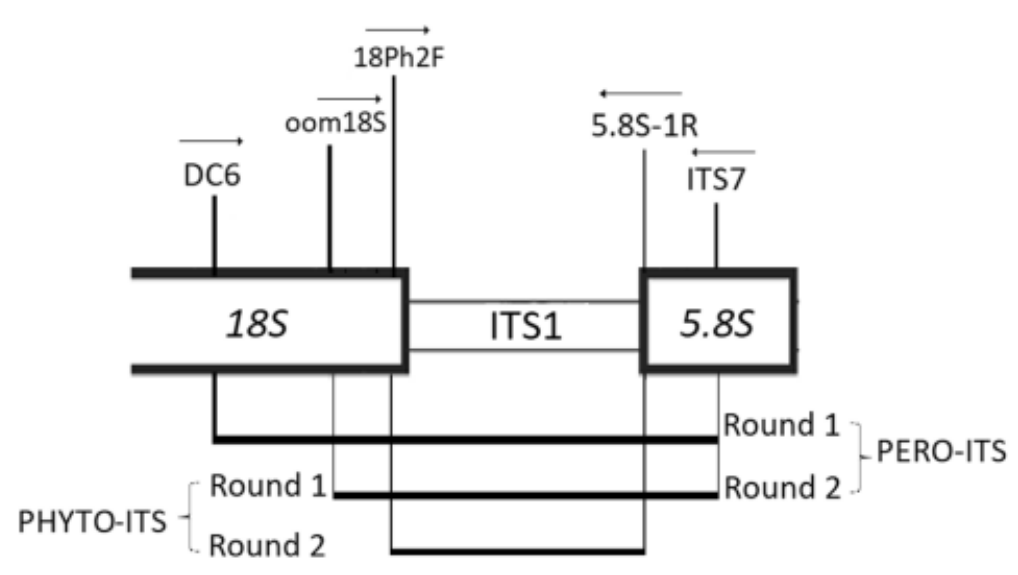

Fig. 1. Map of the 18S, ITS1, and $5.8 \mathrm{~S}$ regions showing positions of different primer pairs

Sequences were sorted and trimmed according to their quality using Mothur (Schloss et al. 2009) and Usearch (Edgar 2010). Only reverse reads (R2) were retained to analyze both ITS sequencing data sets. The forward reads (R1) covered the $18 \mathrm{~S}$ ribosomal DNA region, which is highly conserved and therefore lacks nucleotide polymorphism between Phytophthora species. Furthermore, the paired reads could be used with V3 chemistry $(2 \times 300 \mathrm{pb})$ in future studies, because the average length of the amplified fragment is $480 \mathrm{bp}$. The R2 reads were dereplicated, sorted by decreasing abundance and the singletons were discarded using Usearch "sortbysize" command. Sequences were then clustered with a $99 \%$ similarity threshold in order to discriminate efficiently between species, using Usearch 
cluster_otus command. Molecular Operational Taxonomic Units (OTUs) were taxonomically assigned using BLAST (Altschul at al. 1990) against a local database (all NCBI sequences assigned to Oomycetes with the "ITS" keyword in their GenBank definition), or a homemade database containing Sanger RAS-Ypt sequences from the mock isolates (Table 1). OTUs assigned to the same species were merged according to their taxonomic identity at the species level. Among the 27 samples, ten failed during the Miseq sequencing, because of very low yield or high proportions of contaminants from samples of other studies incorporated into this common Miseq run. The 17 remaining samples were submitted to a random subsampling at 471 reads by sample (minimum depth of the sample set). Because of putative PCR contaminants from other environmental samples in the same HTS run, only OTUs supported by $>10$ sequences were retained in the environmental samples (Miller et al. 2016).
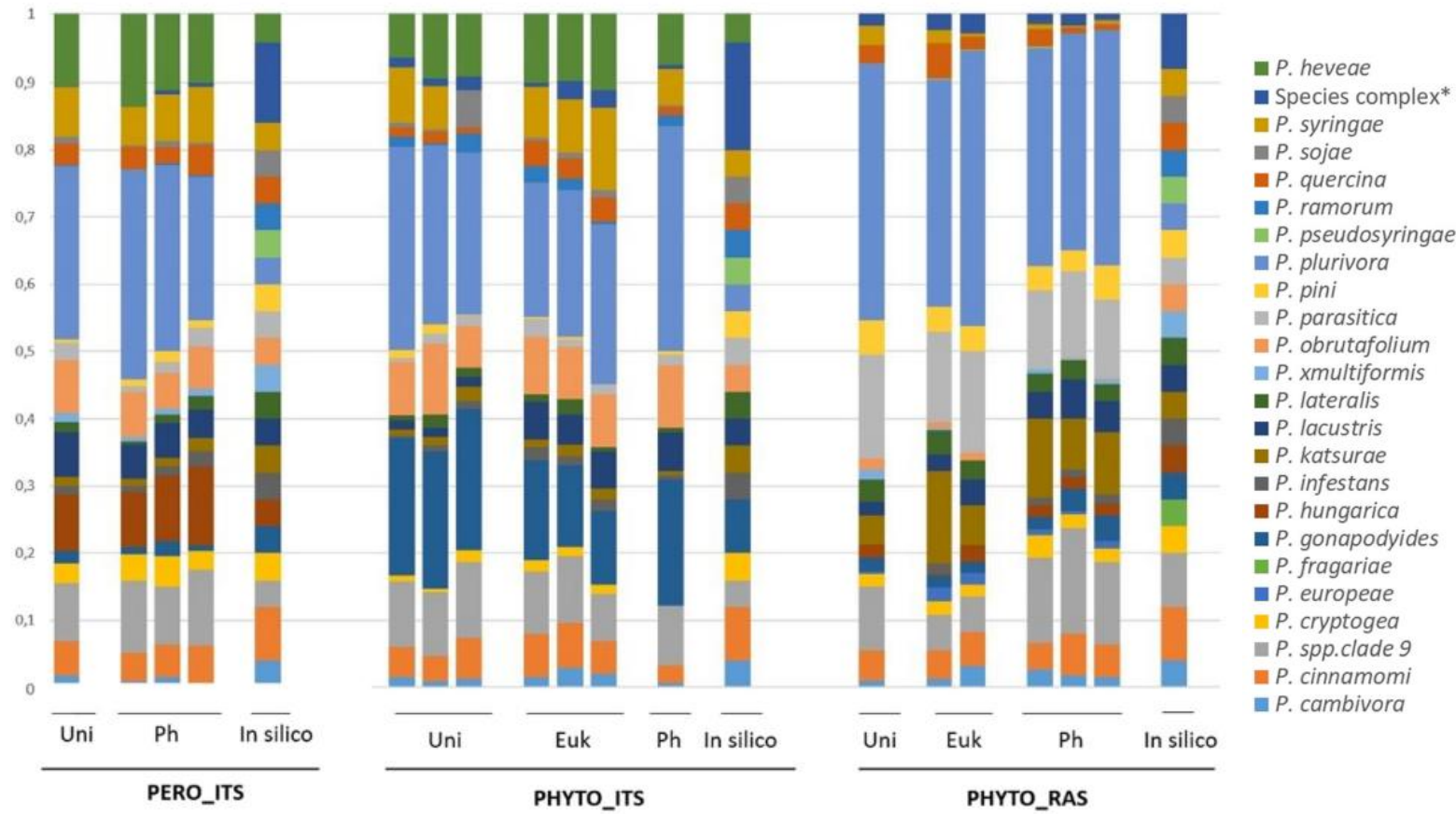

Fig. 2. Proportions of reads per Phytophthora species for each technical replicate, depending on the mock community composition and the PCR amplification strategy. Euk= Eukaryotic mock community; $\mathrm{Ph}=$ Phytophthora-only mock community; Uni = Eukaryotic and prokaryotic mock community. PERO_ITS is the library amplified by Peronosporales-specific primer set oom18S/ITS7; PHYTO_ITS is the library amplified by Phytophthora-specific primer set 18Ph2F/5.8 - 1.5R; RASS_ITS is the library amplified by Phytophthora-specific primer set Ypr1F/Ypr1R. The number of reads by sample was subsampled to 471 reads. *Species complex: represent the fusion of $P$. uniformis, $P$. europeae and $P$. fragariae for PERO_ITS and $P$. uniformis, $P$. europeae, $P$. fragariae and $P$. x multiformis for PHYTO_ITS. "In silico » corresponds to expected OTUs composition based on the clustering at $99 \%$ similarity of 25 Sanger sequences from the RAS or ITS regions. Missing repetitions are due to low quality MiSeq sequencing. Differences in species proportions between samples were tested using the function ADONIS of the $\mathrm{R}$ vegan package (with 99,999 permutations) 
Table 2. Primers pairs used in this study and previous ones. The PHYTO ITS and PERO ITS libraries were created using nested PCR (two consecutive amplifications): first using DC6/ITS7 then oom18S/ITS7 for the PERO_ITS library; first using oom18S/ITS7 then 18Ph2F/5.8S-1R for the PHYTO_ITS library. The PHYTO_RAS library was made using simple PCR

\begin{tabular}{|c|c|c|c|c|c|c|c|}
\hline Primer pair & Region & Specificity & Forward sequence & Reverse sequence & $\begin{array}{l}\text { Anncaling } \\
\text { temperature }\left({ }^{\circ} \mathrm{c}\right)\end{array}$ & Reference & $\begin{array}{l}\text { Used in } \\
\text { this study }\end{array}$ \\
\hline DC6-ITS7 & ITS & Oomycetes & GAGGGACTTTTGGGTAATCA & AGCGTTCTTCACTCGATGTGC & 60 & Cooke et al. $(2000)$ & Yes \\
\hline oom18S-ITS7 & ITS & Peronosporal-es & GCGCATCGTGCTAGGGATAG & AGCGTTCTTCACTCGATGTGC & 60 & $\begin{array}{l}\text { Cordier (designed } \\
\text { for this study) }\end{array}$ & Yes \\
\hline I8Ph2f-5.8SIR & ITS & Phyophthora & GGATAGACTGTTGCATTTTCAGT & GCARRGACTTTCGTCOCYRC & 53 & Scibetta et al. (2012) & Yes \\
\hline YphIF-Yph2R & YPT1 & Phowohthora & CGACCATKGGTGTGGACTTT & ACGTTCTCMCAGGCGTATCT & 58 & Weir et al. (2015) & Yes \\
\hline ITS100-ITS300 & ITS & Oomycetes & $\begin{array}{l}\text { GGAAGGATCATTACCACACGGAAGGATCATTA } \\
\text { CCAC }\end{array}$ & AGTATGYYGTATCAGTG & NA & Riit et al. (2016) & No \\
\hline ITS6-ITS7 & ITS & Oomycetes & GAAGGTGAAGTCGTAACAAGG & AGCGTTCTTCACTCGATGTGC & NA & Cooke et al. (2000) & No \\
\hline
\end{tabular}


Some species with over $99 \%$ similarity in the studied amplicons clustered together. Thus, no more than 20, 22 and 23 species could be retrieved in the PHYTO_ITS, PERO_ITS, and PHYTO_RAS libraries, respectively (Fig. 2). The clustering process generated 75 and 222 Phytophthora OTUs for the PHYTO_ITS and PERO_ITS libraries respectively corresponding to 19 and 21 species, as one species from the original mix. Indeed, as confirmed with in silico analysis, $P$. uniformis, $P$. europeae and $P$. fragariae clustered together at $99 \%$ of similarity for PERO_ITS PCR strategy; and $P$. uniformis, $P$. europeae, $P$. fragariae and P. $\times$ multiformis were identified as one single group at $99 \%$ of similarity also for PHYTO_ITS PCR strategy (Fig. 2). P. pseudosyringae was never identified in both ITS libraries. The RAS amplification gave out 60 different OTUs belonging to 22 species, with $P$. ramorum missing. Proportions of reads from each species were significantly different from the proportions of the original mix regardless of the library $(p<0.001)$. However, proportions were conserved between technical replicates, and did not depend on the added presence of other Dikarya / Procarya DNA in the mock communities $(p=0.99)$. Species distribution patterns were significantly different between each library $(p<0.001)$. Seven different Phytophthora species were detected in environmental samples with the PHYTO_ITS library and just one species ( $P$. syringae) with the PERO_ITS library (Table 3). Non-Phytophthora Oomycetes reads represented $5 \%$ and $98 \%$ of the total reads in the PHYTO_ITS and the PERO_ITS libraries respectively. Phytophthora represent- ed only $2 \%$ of the total reads of PERO_ITS; while 95\% of the reads were assigned to Pythium and Phytopythium and sorted in 117 OTUs pertaining to 29 species. This result constituted $97 \%$ of Peronosporomycete reads with the PERO_ITS amplification. The two nested PCR approaches, DC6/ITS7 x Oom18S/ITS7 (PERO_ITS) and Oom18S/ITS7 × 5.8S1R/18ph2F (PHYTO_ITS), proved reliable for qualitative characterisation of mock Phytophthora communities without other Oomycete species. However, the second PCR strategy was much more effective for environmental samples, usually containing a much higher abundance of other Peronosporaceans such as Pythium (Cerri et al. 2017). Moreover, the number of amplified Phytophthora species corresponded to the order of magnitude of Phytophthora diversity in temperate forests, which is of about 2 to 8 species (Jung et al. 2010; Hansen and Delatour 1999). Thus, our study suggests that the Oom18S/ITS7 x 5.8- S1R/18ph2F primer set is an effective tool for qualitative description of environmental Phytophthora species. Nevertheless, quantitative distortions in the species proportions have been observed in HTS data obtained with the three primer sets, as described with other primers targeting fungal community (Nguyen et al. 2015; Buée et al. 2016). Possible causes include PCR steps and primer affinities for specific species, difference in ITS copy numbers in the genome (Bakker 2018), or errors in initial genomic DNA quantifications. This needs to be taken into account for quantitative studies. On the other hand, no mismatches bias was observed in the Phytophthora mocks supplemented with microbial metagenome background; and 
finally, qualitative detection of Phytophthora species, in order to detect potential pathogenic agents, would be very effective.

Table 3. Number of environmental samples where Phytophthora species were detected, depending on the PCR amplification strategy. A total of 8 soil samples (corresponding to 2 sites $\times 4$ trees) were analyzed. The PHYTO_ITS library was made using the 18Ph2F/5.8S-1R primer pair and the PERO_ITS library using the oom18S/ITS7 primer pair. Retrieved Phytophthora species belonged to 4 clades out of the 10 Phytophthora clades

\begin{tabular}{lccc}
\hline Species & \multicolumn{2}{c}{ Number of samples / library } & Clade \\
\cline { 2 - 3 } & PERO_ITS & PHYTO_ITS & \\
\hline P. cactorum & 0 & 5 & 1 \\
P. europeae & 0 & 3 & 7 \\
P. pini & 0 & 3 & 2 \\
P. plurivora & 0 & 2 & 2 \\
P. pseudosyringae & 0 & 4 & 3 \\
P. sp. neb_1543 & 0 & 1 & 7 \\
P. syringae & 2 & 4 & 8 \\
\hline
\end{tabular}

Although unreliable for the Phytophthora genus, the Peronosporacean-specific primer set enables the coverage of a wider Oomycete community, in particular Pythium and Phytopythium. The amplification of RAS-Ypt region showed good results on mock communities and could represent an alternative marker for barcoding as the ITS region presents low polymorphism in Phytophthora. Furthermore, it has been shown that single-copy markers can provide better relative abundance estimates of fungal taxa than the multi-copy ITS regions (Větrovský et al. 2016; Pérez-Izquierdo et al. 2017). Targeting single-copy marker could therefore improve relative abundance estimates for Phytophthora communities as well, but potentially at a cost of a lower detection threshold. The efficiency of the RAS gene remains to be validated on environmental samples. In conclusion, our study revealed a high concordance between the expected and recovered community composition of Phytophthora within mock communities. Our results show quantitative differences that can be caused by the selection of barcode and associated primer sets. Taken together, these results reveal that "Oom18S/ITS7 $\times 5.8-\mathrm{S} 1 \mathrm{R} / 18 \mathrm{ph} 2 \mathrm{~F}$ " primer set is a robust tool for future qualitative studies of Phytophthora community, richness study and diversity monitoring using the third-generation sequencing generations.

Acknowledgements. We gratefully acknowledge E. Morin for her assistance in bioinformatics advices, and A. Gillet and L. Fauchery for their technical support. We also thank the members of the MetaBAR project ("Meta-omics and microbial ecosystems" INRA Metaprogramme), who provided the non-Phytophthora microbial genomics DNA from INRA microbe collections. We thank also one anonymous 
reviewer for providing helpful comments to improve our manuscript. JL holds $\mathrm{PhD}$ fellow- ships awarded by the "Agence Nationale de la Recherche" as part of the ANR Blanc program NEBEDIV (ANR-13-BSV7-0009) and by the INRA (Metaprogramme MEM). The UMR IAM is supported by a grant overseen by the French National Research Agency (ANR) as part of the "Investissements d'Avenir" program (ANR-11LABX-0002-01, Laboratory of Excellence ARBRE).

\section{References}

Aguayo J, Adams GC, Halkett F, Catal M, Husson C, Nagy ZÁ, Hansen EM, Marçais B, Frey P (2013) Strong genetic differentiation between North American and European populations of Phytophthora alni subsp. uniformis. Phytopathology 103:190-199.

Altschul, S. F., Gish, W., Miller, W., Myers, E. W., \& Lipman, D. J. (1990). Basic local alignment search tool. Journal of molecular biology, 215:403-410.

Bakker MG (2018) A fungal mock community control for amplicon sequencing experiments.

Bellemain E, Carlsen T, Brochmann C, Coissac E, Taberlet P, Kauserud H (2010) ITS as an environmental DNA barcode for fungi: an in silico approach reveals potential PCR biases. BMC Microbiol 10:189.

Bokulich NA, Subramanian S, Faith JJ, Gevers D, Gordon JI, Knight R et al (2013) Qualityfiltering vastly improves diversity estimates from Illumina amplicon sequencing. Nat Methods 10:57.

Brasier CM, Vettraino AM, Chang TTet al (2010) Phytophthora lateralis discovered in an old growth Chamaecyparis forest in Taiwan. Plant Pathol 59:595-603.

Buée M, Sentausa E, Murat C (2016) Molecular technologies applied to the ecology of ectomycorrhizal communities. In: Molecular Mycorrhizal Symbiosis, p 323.

Català S, Pérez-Sierra A, Abad-Campos P (2015) The use of genus-specific amplicon pyrosequencing to assess Phytophthora species diversity using eDNA from soil and water in northern Spain. PLoS ONE 10(3):e0119311.

Cerri M, Sapkota R, Coppi A, Ferri V, Foggi B, Gigante D, Lastrucci L, Selvaggi R, Venanzoni R, Nicolaisen M, Ferranti F, Reale L (2017) Oomycete communities associated with reed die-Back syndrome.Front Plant Sci 8:1550

Coince A, Cael O, Bach C, Lengelle J, Cruaud C, Gavory F, Morin E, Murat C, Marcais B, Buee M (2013) Below-ground fine-scale distribution and soil versus fine root detection of fungal and soil oomycete communities in a French beech forest. Fungal Ecol 6:223 235.

Cooke DEL, Drenth A, Duncan JM, Wagels G, Brasier CM (2000) A molecular phylogeny of Phytophthora and related oomycetes. Fungal Genet Biol 30:17-32.

Edgar, R. C. (2010). Search and clustering orders of magnitude faster than BLAST. Bioinformatics, 26:2460-2461

Egge E, Bittner L, Andersen T, Audic S, de Vargas C, Edvardsen B (2013) 454 pyrosequencing to describe microbial eukaryotic community composition, diversity and relative abundance: a test for marine haptophytes. PLoS One 8(9):e74371.

Hansen E (2008) Alien forest pathogens : Phytophthora species are changing world forests. Boreal Environ Res 13:33-41

Hansen E, Delatour C (1999) Phytophthora species in oak forests of North-East France. Ann For Sci 56:539-547.

Jung T, Blaschke H, Oßwald W (2010) Involvement of soilborne Phytophthora species in Central European oak decline and the effect of location factors on the disease. Plant Pathol 49:706-718. 
Kamoun S, Furzer O, Jones JD, Judelson HS, Ali GS, Dalio RJ, Roy SG, Schena L, Zambounis A, Panabières F, Cahill D, Ruocco M, Figueiredo A, Chen XR, Hulvey J, Stam R, Lamour K, Gijzen M, Tyler BM, Grünwald NJ, Mukhtar MS, Tomé DF, Tör M, Van Den Ackerveken G, McDowell J, Daayf F, Fry WE, Lindqvist-Kreuze H, Meijer HJ, Petre B, Ristaino J, Yoshida K, Birch PR, Govers F (2015) The top 10 oomycete pathogens in molecular plant pathology. Mol Plant Pathol 16:413-434.

Miller KE, Hopkins K, Inward DJ, Vogler AP (2016) Metabarcoding of fungal communities associated with bark beetles. Ecol Evol 6:1590-1600

Nguyen NH, Smith D, Peay K, Kennedy P (2015) Parsing ecological signal from noise in next generation amplicon sequencing. New Phytol 205:1389-1393.

Pérez-Izquierdo L, Morin E, Maurice JP, Martin F, Rincón A, Buée M (2017) A new promising phylogenetic marker to study the diversity of fungal communities: the glycoside hydrolase 63 gene. Mol Ecol Resour 17:e1 - e11

Riit T, Tedersoo L, Drenkhan R, Runno-Paurson E, Kokko H, Anslan S (2016) Oomycetespecific ITS primers for identification and metabarcoding. MycoKeys. 14:17-30.

Sapkota R, Nicolaisen M (2015) An improved high throughput sequencing method for studying oomycete communities. J Microbiol Methods 110:33-39

Schoch CL, Seifert KA, Huhndorf S, Robert V, Spouge JL, Levesque CA,Miller AN (2012) Nuclear ribosomal internal transcribed spacer (ITS) region as a universal DNA barcode marker for Fungi. Proc Natl Acad Sci 109(16):6241-6246.

Schloss, P. D., Westcott, S. L., Ryabin, T., Hall, J. R., Hartmann, M.,Hollister, E. B., et al., Sahl, J. W. (2009). Introducing mothur: open-source, platform-independent, community-supported software for describing and comparing microbial communities. Appl. Environ. Microbiol., 75, 7537-7541.

Scibetta S, Schena L, Chimento A, Cacciola SO, Cooke DEL (2012) A molecular method to assess Phytophthora diversity in environmental samples. J Microbiol Methods 88:356 368.

Taberlet P, Coissac E, Hajibabaei M, Rieseberg LH (2012) Environmental DNA. Mol Ecol 21(8):1789 - 1793.

Taylor DL, Walters WA, Lennon NJ, Bochicchio J, Krohn A, Caporaso JG, Pennanen T (2016) Accurate estimation of fungal diversity and abundance through improved lineage-specific primers optimized for Illumina amplicon sequencing. Appl Environ Microbiol 82:7217-7226.

Tedersoo L, Anslan S, Bahram M, Põlme S, Riit T, Liiv I et al (2015) Shotgun metagenomes and multiple primer pair-barcode combinations of amplicons reveal biases in metabarcoding analyses of fungi. MycoKeys 10:1-43.

Tedersoo L, Tooming-Klunderud A, Anslan S (2018) PacBio metabarcoding of Fungi and other eukaryotes: errors, biases and perspectives. New Phytol 217:1370-1385.

Turner J, O' Neill P, Grant M, Mumford RA, Thwaites R, Studholme DJ (2017) Genome sequences of 12 isolates of the EU1 lineage of Phytophthora ramorum, a fungus-like pathogen that causes extensive damage and mortality to a wide range of trees and other plants. Genomics Data 12:17-21.

Vannini A, Bruni N, Tomassini A, Franceschini S, Vettraino AM (2013) Pyrosequencing of environmental soil samples reveals biodiversity of the Phytophthora resident community in chestnut forests. FEMS Microbiol Ecol 83:433-442.

Větrovský T, Kolařík M, Žifčáková L, Zelenka T, Baldrian P (2016) The rpb2 gene represents a viable alternative molecular marker for the analysis of environmental fungal communities. Mol Ecol Resour 16: 388-401.

Weir B, Paderes E, Anand N, Uchida J, Pennycook S, Bellgard S, Beever R (2015) A taxonomic revision of Phytophthora Clade 5 including two new species, Phytophthora agathidicida and P. cocois. Phytotaxa 205:21-38. 